\title{
Analysis of Effect of Variable Number of Subchannels on the Performance of Filter Bank Multicarrier Prototype Filter
}

\author{
A.S. Kang* and Renu Vig \\ ECE Dept, Panjab University Regional Centre, Hoshiarpur, Punjab, India
}

\begin{abstract}
In the current radio frequency communication system, many spectrum bands are highly utilized while several remain moderately utilized or underutilized. The cognitive radio is a new paradigm to overcome the persisting problem of spectrum underutilization. Seeing the ever increasing demand of wireless applications, the radio spectrum is a precious resource and in cognitive radio systems, trustworthy spectrum sensing techniques are required to be adopted and implemented for the purpose of avoiding any harmful interference to the primary users who have authorized or licensed access to the spectrum. It is up to the secondary users to vacate the channels whenever primary users need the channel. BER and Eb/No are the performance metrics or governing parameters to affect the system performance using polyphase filter bank. The present paper deals with the study of effect of variation of number of subchannels $\mathrm{M}$ at fix overlapping factor $\mathrm{K}$ of polyphase component of Filter Bank Multicarrier cognitive radio in terms of prototype filter length at $\mathrm{L} p=\mathrm{K}^{*} \mathrm{M}-1$ with $\mathrm{K}=4$.
\end{abstract}

Keywords: FBMC; Cognitive-radio; Spectrum; Sub channels; Filter length

\section{Introduction}

The rise in the ever increasing multimedia and data usage applications has led to create immense burden on the wireless service network providers to offer rapid and reliable wireless access. The problem of scarcity of spectrum and congestion in ISM bands can be reduced to some extent by keeping the interference to primary users below a certain threshold. The recent interest in cognitive radio based research has attracted a great deal of attention in spectrum sensing and detection of radio users in the environment. The primary objective is to maximize the probability of detection without losing much on the probability of false alarm while minimizing the complexity and time to sense and detect the radio. The limited spectrum for dense wireless communications and inefficient spectrum utilization necessitates a new communication paradigm cognitive radio which can exploit the unutilized spectrum opportunistically. The evolving research efforts are in the field of cognitive radios covering the different Physical Layer Aspects such as cognitive process, different modulation techniques which are utilised for the signal transmission in $\mathrm{CR}$ and varying spectrum sensing techniques. CR as a future wireless communication system is characterized as a system, which is able to adapt its transmission and reception parameters on the basis of cognitive interaction with the wireless environment in which it actually operates. There are several reasons that make spectrum sensing, a practically challenging task. A very low required SNR for primary user detection, multi-path fading and time dispersion effects of wireless channels which tend to complicate the spectrum sensing problem and the frequently changing noise level with time and location causes the noise power uncertainty a big issue of concern for primary user detection [1].

\section{Physical layer: spectrum management}

Spectrum sensing is the main task of the Physical layer of a cognitive radio. Cognitive radio reflects the situations where both primary and secondary users occupy the same channel space as in licensed band situations. It is also responsible for spectrum sensing and reconfiguration of the transmission parameters. Cognitive Radio can reconfigure its operating frequency, modulation, channel coding and output power without hardware replacement, this is the most significant difference between cognitive radio network and other wireless networks physical layer [2-4]. Software Defined Radio (SDR) based RF front-end transmitters and receivers are required for configurability of cognitive radio networks. Implementing RF front-end, heavy-weight signal processing algorithms, detecting weak signals, presence of PU while there are secondary users, are significant sensing problems in Cognitive Radio [1].

\section{Main features of physical layer}

PHY Transport: 802.22 use Orthogonal Frequency Division Multiplexing (OFDM) as transport mechanism. Modulation: QPSK, 16-QAM and 64-QAM are supported. Coding: Convolutional Code is Mandatory. Turbo, LDPC or Shortened Block Turbo Codes are optional but recommended. Pilot Pattern: Each OFDM/OFDMA symbol is divided into sub-channels of 28 sub-carriers of which 4 are pilots, which are inserted every 7 sub-carriers. No frequency domain interpolation is required. Net Spectral Efficiency: 0.624 bits $/ \mathrm{s} / \mathrm{Hz}$ -3.12 bits/s/Hz. Spectral Mask: 802.22 have adopted the Spectral Mask requirements proposed by FCC. (200 tap FIR filter required). Figure 1 shows the Block diagram of CR Model showing the importance of Physical layer.

\section{Earlier Related Work}

Ball and Ferguson [2] have focussed on the potential of CR in military and emergency service uses. Some of the issues involved in adapting cognitive radio technology consumer markets were examined and some potential advantages to CR defined networks

*Corresponding author: A.S. Kang, ECE Dept, Panjab University Regional Centre Hoshiarpur, Punjab, India, E-mail: askang_85@yahoo.co.in

Received January 08, 2014; Accepted February 20, 2014; Published February 24,2014

Citation: Kang AS, Vig R (2014) Analysis of Effect of Variable Number of Subchannels on the Performance of Filter Bank Multicarrier Prototype Filter. J Electr Electron Syst 3: 121. doi:10.4172/2332-0796.1000121

Copyright: (C) 2014 Kang AS, et al. This is an open-access article distributed under the terms of the Creative Commons Attribution License, which permits unrestricted use, distribution, and reproduction in any medium, provided the original author and source are credited. 


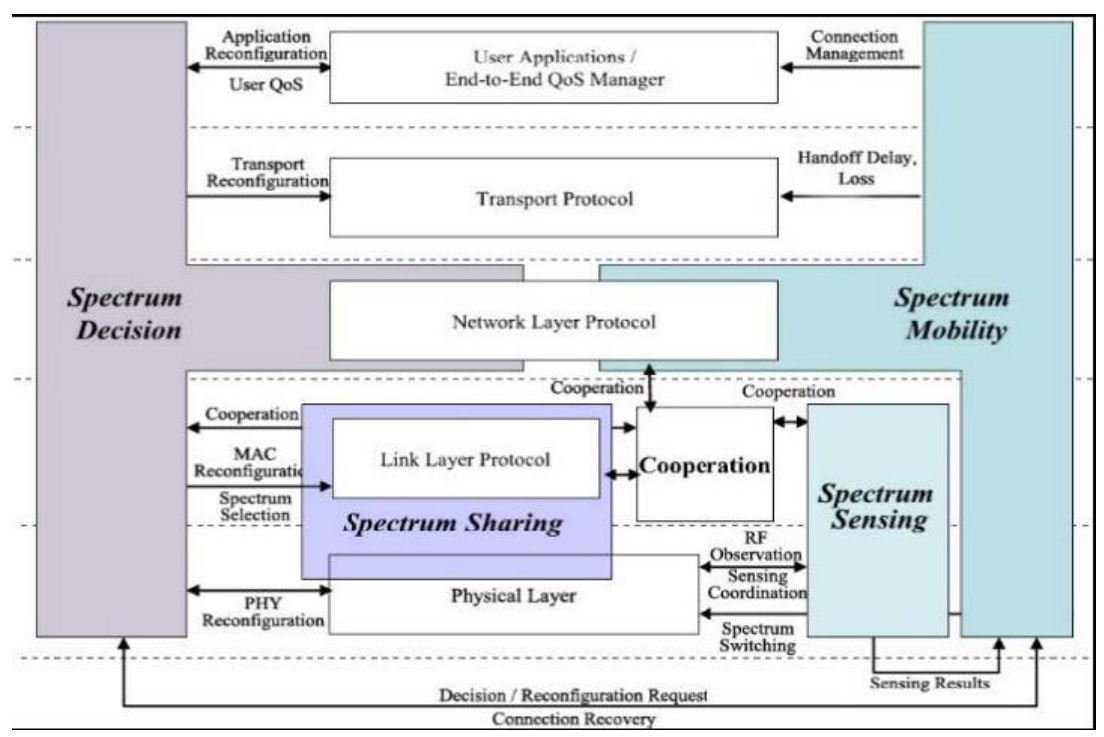

Figure 1: CR Block Diagram of OSI Model.

for consumers were highlighted with some possible service designs and pricing systems. Ihalainen et al. [3] introduced a new low complexity per-subcarrier channel equalizer for FBMC transceiver for high-rate wide band communication over doubly-dispersive channel and analyzed its performance. It was shown that the coded error-rate performance of FBMC is somewhat better than that of the OFDM reference. Zhang et al. [4] discussed that benefits of CR are clear when used in emergency situations. The idea of applying CR to emergency network is to alleviate this spectrum shortage problem by dynamically accessing free spectrum resources.CR is able to work in different frequency bands and various wireless channels and supports multimedia services such as voice, data and video. Reconfigurable radio architecture is proposed to enable the evolution from the traditional Software Defined Radio (SDR) to CR. Liu [5] investigated the motive, definition and paradigms of cognitive radio. The author described the system model used comprising of one primary user and one secondary user with perfect channel side information available at both its transmitter and receiver and channel fading gains. Kang et al. [6] studied the optimal power control policies for fading channels in cognitive radio networks considering both the transmit and interference power constraints. For each of the constraints peak power and average power are investigated. The author derived the optimal power allocation strategies in terms of maximizing the ergodic capacity of the secondary user when channel state information is available to the transmitter and the receiver. Shaat and Bader [7] addressed the problem of resource allocation in multicarrier based CR networks. The objective is to maximize the downlink capacity of the network under both total power and interference introduced to the primary users constraints. The optimal solution has high computational complexity, which makes it unsuitable for practical applications and hence a low complexity suboptimal solution is proposed. The performance of using FBMC instead of OFDM in CR systems is investigated. Simulation results show that the proposed resource allocation algorithm with low computational complexity achieves near optimal performance and proves the efficiency of using FBMC in CR context. Peng et al. [8] discussed the analysis and simulation of sensing deception in fading cognitive radio networks.
Celebi et al. [9] proposed cognitive radio implementation by using standard wireless communication laboratory equipments such as signal generator and spectrum analyzer. Equipments are controlled through MATLAB instrument control toolbox to carry out CR capabilities specified by IEEE 802.22 WRAN standard. The aim of the work is to provide a CR environment for spectrum sensing algorithms to perform a comparative study considering wireless microphone signals for research and educational purposes. Wang et al. [10] discussed the cognitive radio simulation environment realization based on autonomic communication. Virk and Sharma [11] analysed the BER performance of Cognitive Radio Physical Layer over Rayleigh channel under different channel encoding schemes, digital modulation schemes and channel conditions. It has been anticipated from simulation study that the performance of the communication system degrades with the increase of noise power. Shaat and Bader [12] considered a multicarrier based CR network. The goal is to maximize the total sum rate of CR system while ensuring that no excessive interference is induced to the primary system. Kaur [13] studied the wireless networks with cognitive radio technology and discussed its various functions and capabilities as a new networking paradigm for future wireless communication.

\section{Problem Formulation}

Large parts of assigned spectrum are underutilized while the increasing number of wireless multimedia applications led to spectrum scarcity. Cognitive radio is an option to utilize non used parts of the spectrum that actually are assigned to primary services. The benefits of cognitive radio are clear in the emergency situations. Current emergency services rely much on the public networks. This is not reliable in public networks where the public networks get overloaded. The major limitation of emergency network needs a lot of radio resources. The idea of applying Cognitive Radio to the emergency network is to alleviate this spectrum shortage problem by dynamically accessing free spectrum resources. Cognitive Radio is able to work in different frequency bands and various wireless channels and supports multimedia services such as voice, data and video. The literature survey shows that the performance of FBMC based CR system can be enhanced under different radio environment. 
There is a need to work out on the following gaps that have been affecting the performance of cognitive radio.

a) The spectral efficiency of OFDM based Cognitive Radio is less as compared to FBMC based CR due to the insertion of Cyclic Prefix in OFDM results in high spectral leakage in its prototype filter. FBMC is severely affected by non linear distortions but the resulting out of channel leakage is still lower compared to OFDM. Hence, non linear distortion must be minimized for efficient FBMC systems.

b) The problem of vertical and horizontal sharing of radio spectrum and transmission power control exists.

c) The problem of Interference in radio environment under different nodes for primary and secondary user locations results in spectrum underutilization.

d) Optimization and trade-off of power, capacity constraints variables under CR radio environment should be considered.

e) The Resource Allocation Strategies to combat inherent interference on primary user are less efficient.

f) The performance of CR in terms of Energy Spectrogram and Power Spectrogram under different modulation and coding techniques in different radio environment (fading channels) without picocell, microcell, femtocell approach with different indoor and outdoor propagation models using spectrum sensing approach is not effective.

g) Lack of Interlayer Optimization (Cross Layer design) results in degradation in radio resource management.

h) The problem of Congestion in ISM bands adversely affecting the quality of communication persist in Conventional OFDM based network without Dynamic Spectrum Access which

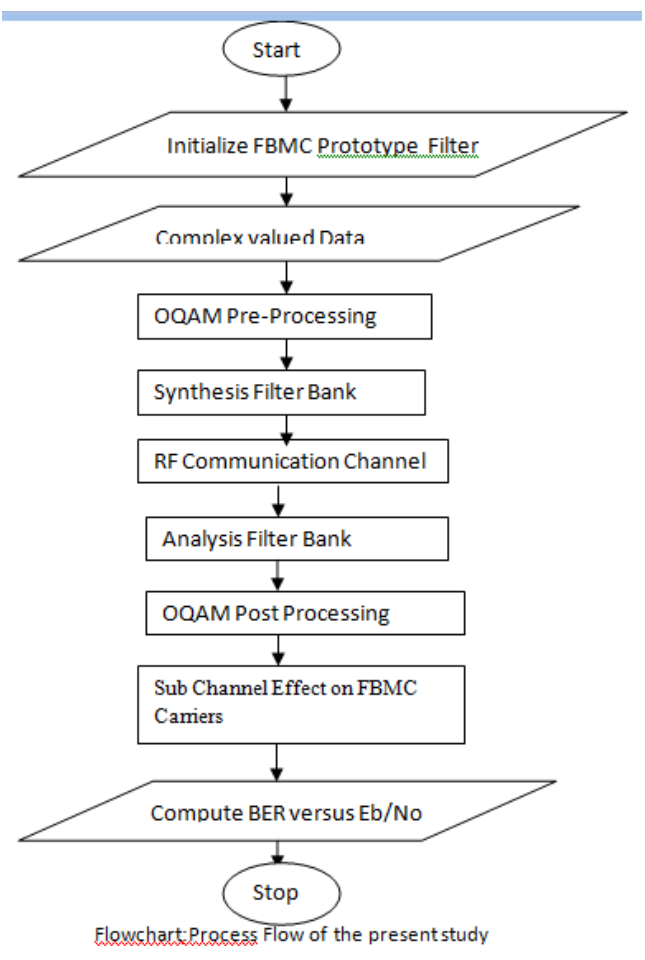

Figure 2: Flowchart for the Process Flow. is not there in CR based network (with dynamic spectrum access).

The present study puts its focus on Performance Enhancement of Filter Bank Multicarrier (FBMC) based Cognitive Radio (CR) in adaptive, opportunistic, autonomic domain under different strategic conditions of wireless environment. By introducing techniques to improve the spectral efficiency and minimizing the spectrum underutilization, hence improving the overall performance of FBMC based CR

\section{Flowchart for Present Study}

A flowchart has been prepared for study of effect of sub channels on prototype filter length for FBMC cognitive radio. The following system parameters have been used in the present investigation. K-Overlapping Factor, M-Number of Subchannels, D=Channel Delay, Lp=Prototype Filter Length. The distinctive feature of the FBMC design technique has ability to provide improved frequency selectivity through the use of longer and spectrally well shaped prototype filters. In the present case, more emphasis has been laid on the Lp=KM-1-D as a specific prototype filter length, under the assumption of Channel Delay D as zero (Figure 2).

\section{Results and Discussion}

Different Graphic plots have been obtained between the various parameters namely Number of Subchannels M, Bit Error Rate BER, Signal to Noise Ratio SNR, Prototype Filter Length $L p=K^{\star} M+1$ at a fix value of Overlapping factor $\mathrm{K}=4$. The spectrum sensing is performed by measuring the signal strength at the outputs of the subcarrier channels at the receiver. Multitaper Spectrum Sensing technique is a fourier transform of slepian vectors which have maximal energy concentration in the bandwidth of $\mathrm{Fc}-\mathrm{W}$ to $\mathrm{Fc}+\mathrm{W}$ under a finite sample size constraint. Filter Bank based Spectrum Estimation is regarded as a simplified version of Multitaper Spectrum Estimation (MTSE) which uses only one prototype filter for each band is used for multicarrier modulation based Cognitive radio systems.CR user identifies the spectrum occupancy and hence the spectrum opportunities. MTSE is better for small samples whereas filter bank based spectrum sensing is better for large number of samples. The Cognitive Radio system is able to transmit over the direct link more than that when the direct link is blocked for all subcarriers in the source side. The impact of the present study of FBMC CR is highlighted through the role of number of subchannels. Readjustment of various parameter levels leads to optimization between different radio environment parameters under varying strategic conditions. The computational complexity of the FBMC cognitive radio is studied under the effect of K, M and Lp. For FBMC system, the prototype coefficients are assumed to be equal to PHYDAS coefficients with overlapping factor $\mathrm{K}=4$.Actually, the entire process here involves the three steps: fixing the subcarriers, matching the subcarriers and readjusting the assigned subcarriers as per the system requirement on an average basis. For optimization and trade-off sake, the number of subcarriers is taken to be greater than 8.The literature survey on FBMC shows that the different subcarriers are adjusted in such a way that the interference to the primary user by secondary users is kept to a minimum. Moreover, the impact of different constraints values on the system performance is investigated. This paper describes how to select and match some subcarriers for transmission and use the rest only for direct transmission. The FBMC CR in physical layer is a potential candidate for future wireless communication system. A Bandwidth of $10 \mathrm{Mhz}$ with $\mathrm{M}=64,128,256,512$ subcarriers have been 
Citation: Kang AS, Vig R (2014) Analysis of Effect of Variable Number of Subchannels on the Performance of Filter Bank Multicarrier Prototype Filter. J Electr Electron Syst 3: 121. doi:10.4172/2332-0796.1000121

Page 4 of 7

taken into consideration in the present scenario. The subcarriers are allocated sequentially to the users with optimum results. In Figure 3 BER is found to decrease and increase at different values of SNR .A staircase plot between Lp and BER has also been obtained. A loglog and semilog plots between various performance parameters have been depicted. It has been found that with increasing BER, SNR is found to decrease and vice versa. SNR range can be taken from $-5 \mathrm{db}$ to $+30 \mathrm{db}$ as per IEEE 802.22 standard recommendations for cognitive radio. Here the suitable SNR range has been taken from 0 till $5 \mathrm{~dB}$ and following results have been found for following different cases of $\mathrm{M}$ (Figures 4-9).

\section{Case1: $\mathrm{M}=64$}

$\mathrm{BER}=1.4$ at $\mathrm{SNR}=0 \mathrm{db}, \mathrm{BER}=1$ for $\mathrm{SNR}=1 \mathrm{db}, \mathrm{BER}=0.75$ at
$\mathrm{SNR}=2 \mathrm{db}, \mathrm{BER}=0.38$ at $\mathrm{SNR}=3 \mathrm{db}, \mathrm{BER}=0.25$ at $\mathrm{SNR}=4 \mathrm{db}, \mathrm{BER}=0.16$ at $\mathrm{SNR}=5 \mathrm{db}$.

\section{Case2: $\mathrm{M}=128$}

$\mathrm{BER}=1.57$ at $\mathrm{SNR}=0 \mathrm{db}, \mathrm{BER}=1$ at $\mathrm{SNR}=1 \mathrm{db}, \mathrm{BER}=0.65$ at $\mathrm{SNR}=2 \mathrm{db}, \mathrm{BER}=0.7$ at $\mathrm{SNR}=3 \mathrm{db}, \mathrm{BER}=0.25$ at $\mathrm{SNR}=4 \mathrm{db}, \mathrm{BER}=0.1$ at $\mathrm{SNR}=5 \mathrm{db}$

Case3: $\mathrm{M}=256$

$\mathrm{BER}=1.6$ at $\mathrm{SNR}=0 \mathrm{db}, \mathrm{BER}=1.17$ at $\mathrm{SNR}=1 \mathrm{db}, \mathrm{BER}=0.55$ at $\mathrm{SNR}=2 \mathrm{db}, \mathrm{BER}=0.5$ at $\mathrm{SNR}=3 \mathrm{db}, \mathrm{BER}=0.25$ at $\mathrm{SNR}=4 \mathrm{db}, \mathrm{BER}=0.2$ at $\mathrm{SNR}=5 \mathrm{db}$

Case $4: \mathrm{M}=512$

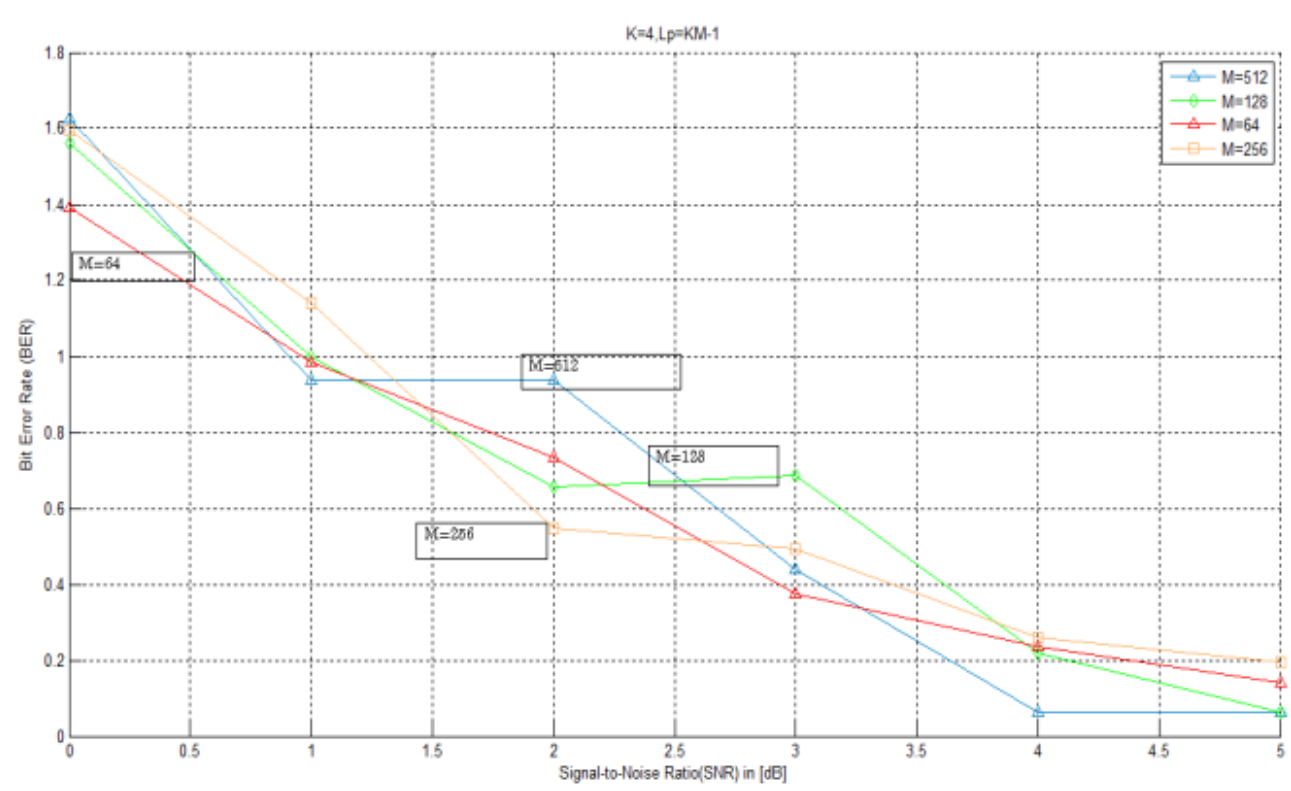

Figure 3: $B E R$ versus $S N R$ plot at $K=4, L p=K{ }^{\star} M-1$.

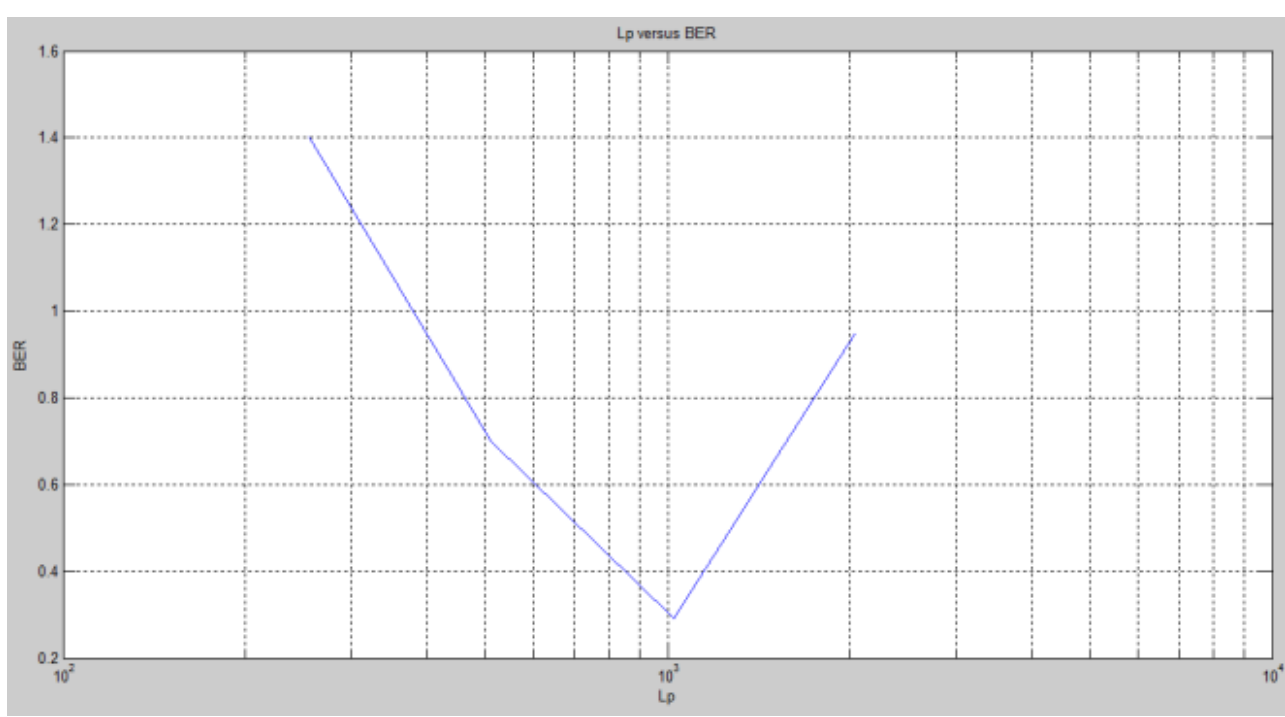

Figure 4: Matlab plot between Lp and BER

BER decreases and then again increases for different values of Prototype filter length Lp beyond $10^{3}$. 


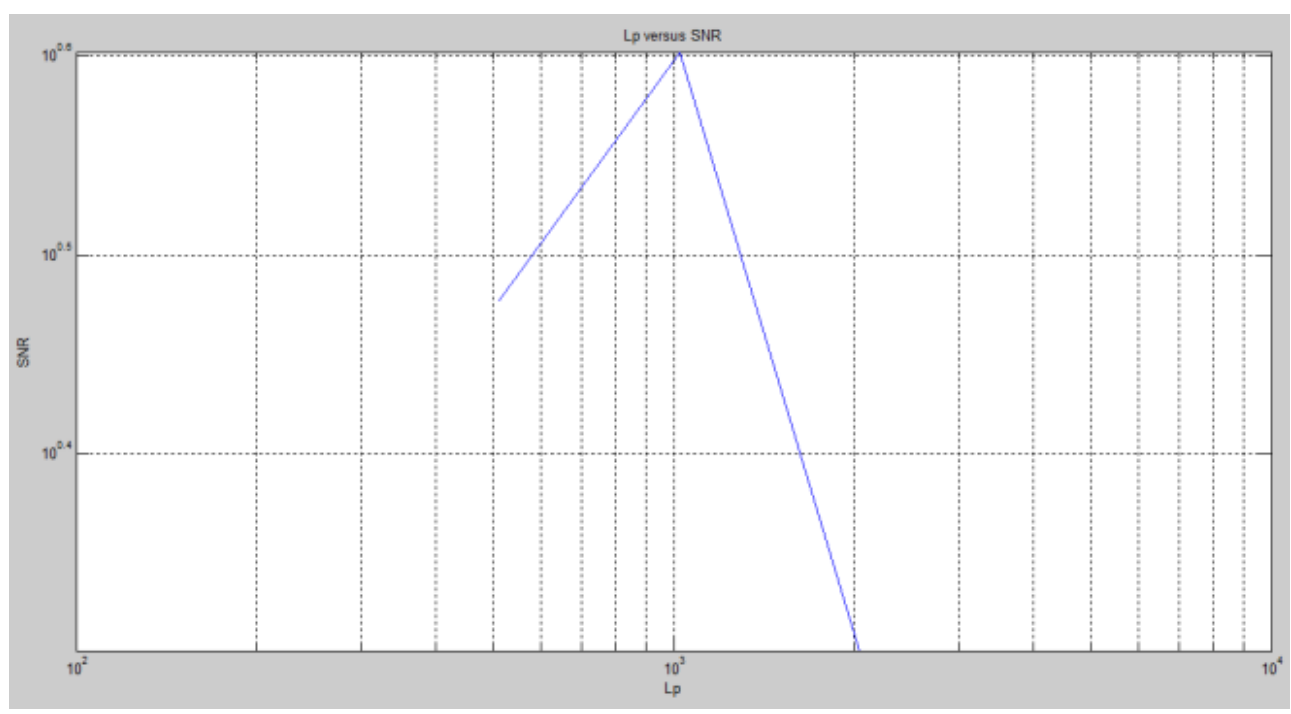

Figure 5: Matlab Plot between Lp and SNR

With increasing SNR, BER is found to increase till $L p=10^{3}$ after which fall in the SNR is noticed with increase in $L p$.

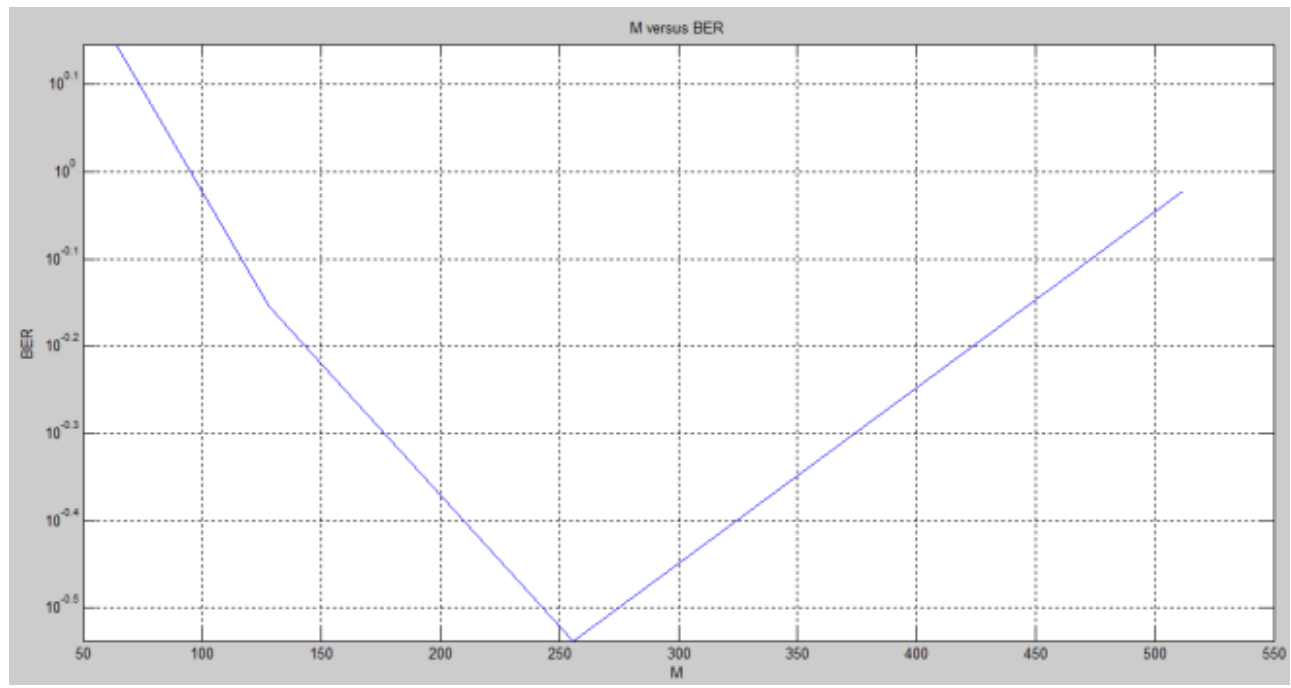

Figure 6: Matlab plot between $M$ versus BER

BER decreases with increasing $M$ but beyond $M=256$, BER again increases to $10^{-0.5}$

$\mathrm{BER}=1.6$ at $\mathrm{SNR}=0 \mathrm{db}, \mathrm{BER}=0.95$ at $\mathrm{SNR}=1 \mathrm{db}, \mathrm{BER}=0.95$ at $\mathrm{SNR}=2 \mathrm{db}, \mathrm{BER}=0.45$ at $\mathrm{SNR}=3 \mathrm{db}, \mathrm{BER}=0.08$ at $\mathrm{SNR}=4 \mathrm{db}, \mathrm{BER}=0.08$ at $\mathrm{SNR}=5 \mathrm{db}$

\section{Conclusion}

Cognitive radio networks have a promising future and have excellent applications of wireless networks. The adaptive technology naturally presents unique signal processing challenges in cognitive radio domain. The signal processing prospective of CR has significant impact in CR technology in its performance enhancement. The use of allocated spectrum varies at different times and over different geographical regions. To overcome the spectrum deficiencies and inefficient utilization of allocated frequencies, it is mandatory to take into consideration the latest communication models by means of which radio frequency spectrum can be utilized wherever the void or white space is available. The present paper shows the comparatative analysis of number of subchannels $\mathrm{M}$ on FBMC prototype filter length at fix overlapping factor $\mathrm{K}=4$ using $\mathrm{BER}$ and $\mathrm{Eb} / \mathrm{No}$ as performance measuring indicators. Other matlab plots have been obtained between $L p$ versus $B E R, L p$ versus $S N R, M$ versus $B E R, M$ versus $L p$, $M$ versus SNR. So, an attempt has been made to analyse the impact of parameters: K, Lp, M and BER on signal impairments in the FBMC based cognitive radio.

\section{Impact of Study}

The expected outcome of proposed research will be helpful in detecting interference at primary receiver and speed and reliability of detection in Cognitive cycle of CR system. The Spread Spectrum Detection in terms of Power and with wide frequency range hidden in the noise can be done. The hidden node problem due to the 


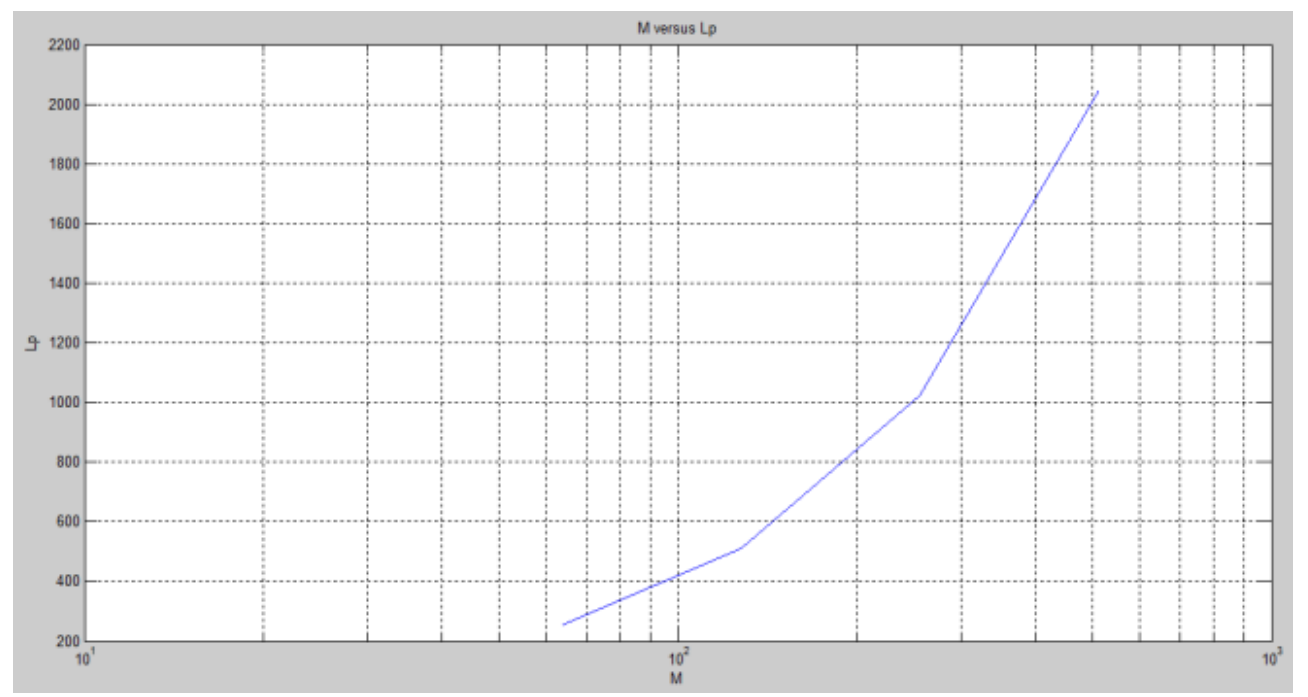

Figure 7: Matlab plot between $M$ and Lp

With increasing $M$, the $L p$ is found to increase at different instants.

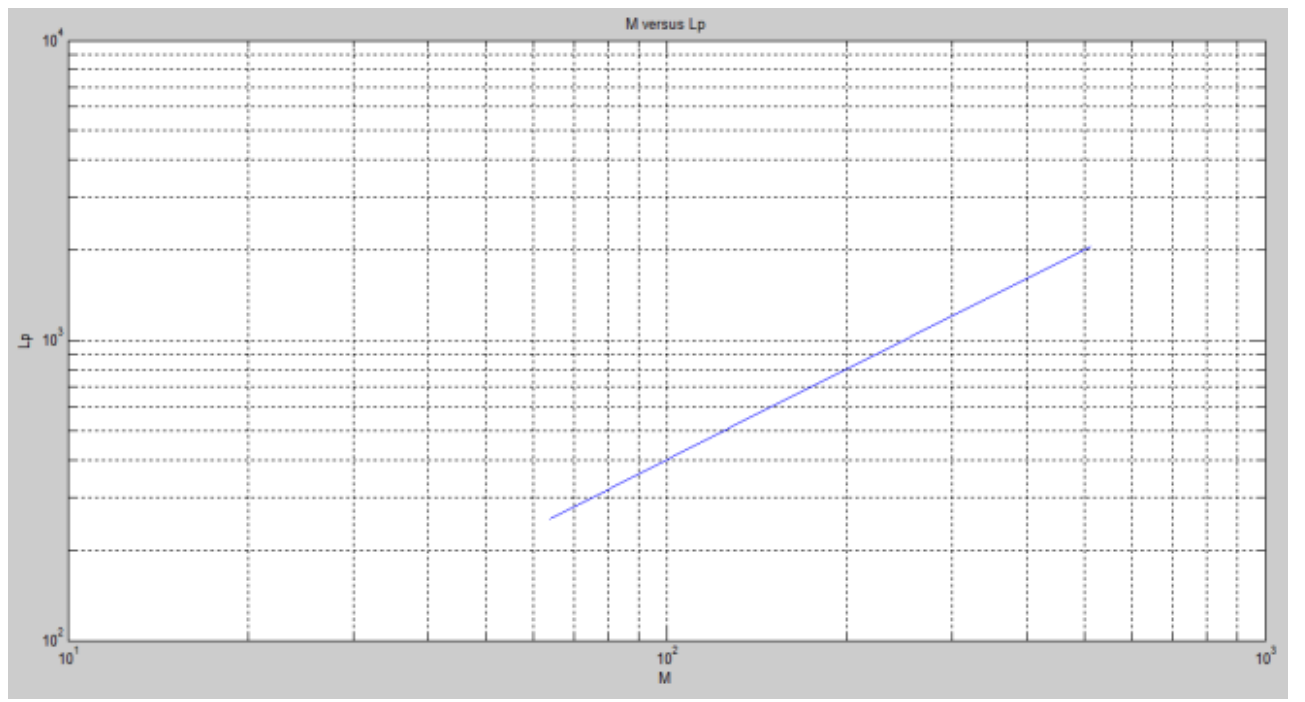

Figure 8: Matlab plot between $M$ and $L p$

With increasing number of Subchannels $M, L p$ is found to increase.

multipath fading in the propagation between primary transmitter and sensing receiver can be studied by applying learning and intelligence approach. The most of the CR will have to autonomously work in multiservice, multi technology and multiuser environment and it has to adapt the different parameters under different conditions. Vertical and Horizontal sharing of radio spectrum will be possible to some extent with efficient spectrum space opportunities, spectrum mobility and transmission power control. The CR must be capable of spectrum sensing and operating over wide radio spectrum range, emulate many radio technologies and different modulation schemes which cause various hardware challenges. The present study will be useful for optimum selection of hardware components which will minimize circuit complexity and cost and less chances of interference. OFDM based CR network performance will improve with the application of multirate signal processing in multicarrier wireless communication.
Better Radio Resource Management is possible. The problem of Congestion in ISM bands which adversely affects the quality of communication will be reduced by CR networks based on Dynamic Spectrum Access.

\section{Future Directions}

As Cognitive radio technology is an important innovation for the future of communications and likely to be a part of the new wireless standards, becoming almost a necessity for situations with large traffic and interoperability concerns. Moreover CR is devised to be used with telecommunications or computer network related disciplines but there are inadequate facilities to provide robustness and effective security. To overcome this, existing technologies will increase the complexity and new types of attack are possible. Therefore, innovative ideas are required to provide security to Cognitive Radio Networks 
Citation: Kang AS, Vig R (2014) Analysis of Effect of Variable Number of Subchannels on the Performance of Filter Bank Multicarrier Prototype Filter. J Electr Electron Syst 3: 121. doi:10.4172/2332-0796.1000121

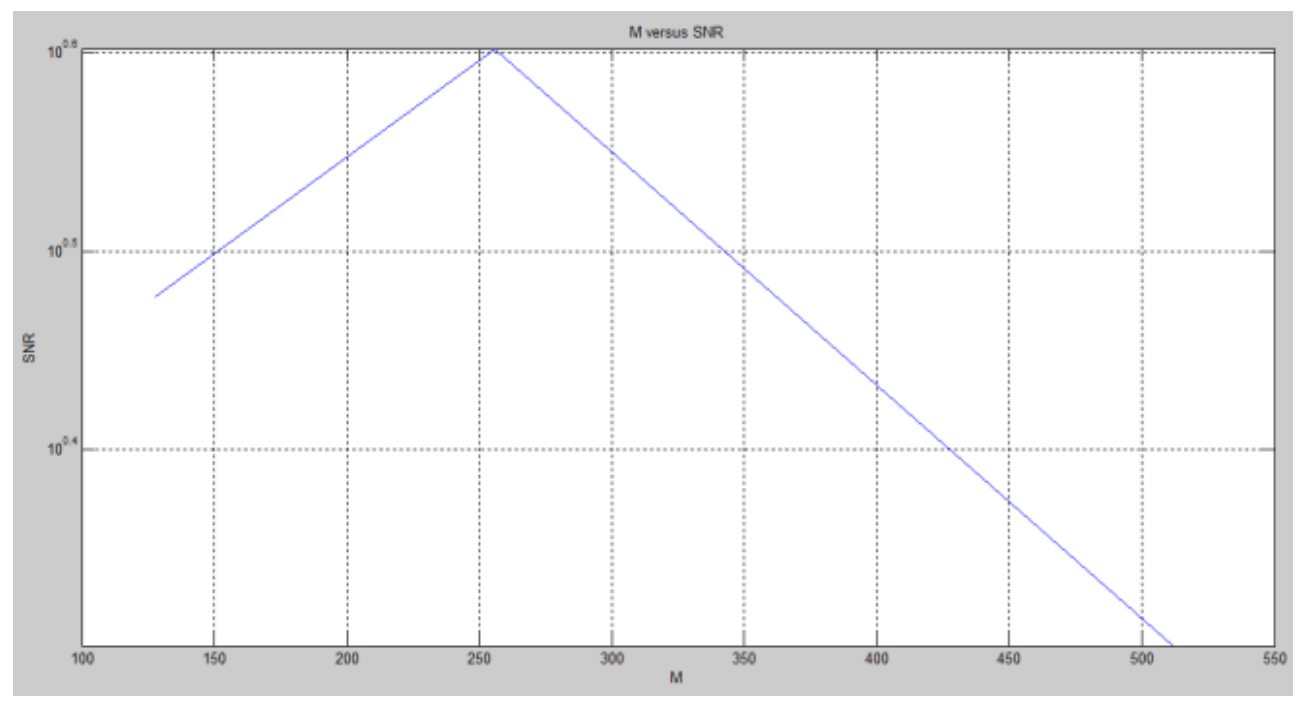

Figure 9: Matlab plot between $\mathrm{M}$ and SNR

$S N R=10^{0.6}$ for $M<250$ but beyond $M=250$, SNR is found to decrease.

and make them robust against crucial attacks, especially the attacks inherent to the Cognitive Radio functionality. So to make Cognitive Radio systems trustworthy, dependable and efficient, a comprehensive energy efficient mechanism is required to identify, remove or mitigate the attacks at any phases of the Cognitive Cycle.

\section{References}

1. Mitola J, Maguire GQ (1999) Cognitive Radios: Making Software Radios More Personal. IEEE Pers Commun 6: 13-18.

2. Ball S, Ferguson A (2005) Consumer Applications of Cognitive Radio Defined Networks. First IEEE International Symposium on New Frontiers in Dynamic Spectrum Access Networks, Baltimore, MD, USA.

3. Ihalainen T, Stitz TH, Rinne M, Renfors M (2007) Channel Equalization in Filter Bank based Multi carrier Modulation For Wireless Communications. EURASIP Journal on Advances in Signal Processing 2007: 1-18.

4. Zhang Q, Hoeksema FW, Kokkeler ABJ, Smit GJM (2010) Towards Cognitive Radio for Emergency Networks. Mobile Multimedia: Communication Engineering Perspective. Nova Publishers, USA.

5. Liu S (2008) Capacity Comparison for Fading Channels under Average and Peak Interference Power Constraints in Underlay Cognitive Radio System.

6. Kang X, Liang Y-C, Nallanathan A (2008) Optimal Power Allocation for Fading Channels in Cognitive Radio Networks under Transmit and Interference Power Constraints. Proc IEEE International Conference on Communications, Beijing, China.

7. Shaat M, Bader F (2010) Computationally Efficient Power Allocation Algorithm in Multicarrier- Based Cognitive Radio Networks, OFDM and FBMC systems. EURASIP Journal on Advances in Signal Processing 2010: 1-13.

8. Peng Q, Cosman PC, Milstein LB (2010) Analysis and Simulation of Sensing Deception in Fading Cognitive Radio Networks. $6^{\text {th }}$ International Conference on Wireless Communications Networking and Mobile Computing (WiCOM), Chengdu, China.
9. Celebi MB, Celebi H, Arslan H, Qaraqe K (2010) Spectrum Sensing Testbed Design for Cognitive Radio Applications. IEEE 19 ${ }^{\text {th }}$ Conference on Signal Processing and Communications Applications (SIU), Antalya, Turkey.

10. Wang S, Liu H, Xie L, Hu W (2011) Cognitive Radio Simulation Environment Realization based on Autonomic Communication. Proc IEEE $3^{\text {rd }}$ International Conference on Communication Software and Networks, Xi'an, China.

11. Virk AK, Sharma AK (2011) BER Performance Analysis of Cognitive Radio Physical Layer over Rayleigh fading channel. Int J Comp Appl 25: 25-29.

12. Shaat M, Bader F (2012) Comparison of OFDM and FBMC Performance in Multi-Relay Cognitive Radio Network. Proc International Symposium on Wireless Communication Systems, Paris, France.

13. Kaur S (2013) Intelligence in Wireless Networks with Cognitive Radio Networks! IETE Technical Review 30: 6-11. 